\title{
EXAME NEUROLÓGICO EVOLUTIVO E DIFICULDADE NO APRENDIZADO
}

\author{
Newra Tellechea Rotta *
}

\begin{abstract}
A importância do exame evolutivo na avaliação neurológica da criança é um tema sem controvérsias, já que todos estão de acordo em que a mesma medida não pode ser usada para o adulto e para a criança. Um ser em pleno desenvolvimento necessita medidas diferentes para cada estágio que atravessa. Como uma resposta às dificuldades que o neuropediatra encontrava para avaliar as funções motoras e perceptivas da criança, surgiu, em 1972, a publicação sobre Exame Neurológico Evolutivo dos 3 aos 7 anos de idade, fruto de um trabalho coordenado pelo Prof. Lefèvre ", em São Paulo, e do qual participaram vários especialistas.
\end{abstract}

A partir desse momento passamos a aplicar o método, principalmente na avaliação neurológica das crianças que cursavam grupos escolares sob a responsabilidade da Secretaria de Educação e Cultura do Estado do Rio Grande do Sul, e iniciamos esta pesquisa prospectiva onde procuramos relacionar os achados do exame neurológico evolutivo com as dificuldades do aprendizado.

\section{MATERIAL E METODOS}

Foram selecionadas 100 crlanças, distribuidas em dois grupos: grupo A (50 criancas com bom rendimento escolar) e grupo B (50 criancas com dificuldades escolaras). Estes dois grupos loram separados de uma população de crianças que, em 1973, cursou a $1^{\text {H }}$ série do $1^{\circ}$ ciclo em grupos escolares considerados como mostragem da população escolar do Estado do Rio Grande do Sul.

Foram selecionadas para o grupo A as criansas que, tendo cursado a $1^{\text {a }}$ série em 1973, obtiveram aprovação para a $2^{n}$ série sem terem apresentado dificuldades escolares, seja relacionadas com a leitura, a escrita ou o cálculo.

Foram reunidas no grupo $B$ as crianças que em 1973 apresentaram dificuldades relacionadas com a escrita, a leitura ou o cálculo e, em 1974, ou foram aprovadas para a $2^{\prime}$ série após atendimento especial (aí incluindo classe de recuperação e clinicas de leitura), e que na $2^{*}$ série também estão apresentando problemas, ou permaneceram na $1^{\text {n }}$ série, ou foram encaminhadas para classe especial.

Os dois grupos foram submetidos a exame neurológico tradicional e não apresentaram anormalidades, a não ser hipotonia muscular.

Relatório aprescntado no VI Congresso Brasileiro de Neurologia, junho de 1974: * Prof. Auxiliar de Neurologia na Faculdade de Medicina de Porto Alegre e na Fundação Faculdade Católica de Medicina de Porto Alegre; responsável pelo setor de neuropediatria do Instituto de Neurologia da Santa Casa de Misericórdia de Porto Alegre e pelo Setor de Neurologia da Secretaria de Educacão e Cultura do Rio Grande do Sul. 
Trabalharam nesta pesquisa duas psicólogas e todas as crianças foram submetidas ás baterias de testes de Bender e WISC, com o que se quis fazer o estudo comparativo dos achados neurológicos e psicológicos e valorizar o trabalho em equipe.

As crianças vieram para a investigação neurológica sem que se tivesse conhecimento do grau de aproveitamento escolar. Todas foram submetidas ao exame neurológico evolutivo (ENE) e, embora as idades variassem entre 7 e 10 anos, foi possivel observar crianças com mais de 7 anos com impossibilidade para realizar provas do ENE.

Foram relacionados como iguais ou superiores a 7 anos aqueles casos em que a criança teve condições para realizar satisfatòriamente as provas para esta idade, independente da sua idade cronológica. $E$ evidente que, se tivéssemos medidas para 8 , 9 e 10 anos, alguns casos do grupo B que constam como tendo apresentado ENE normal, não fariam parte deste grupo e a percentagem de $14 \%$ de normalidade aí encontrada decresceria.

Do grupo A fazem parte 24 crianças do sexo masculino e 26 do sexo feminino. Do grupo B fazem parte 27 crianças do sexo masculino e 23 do sexo feminino. As 50 crianças do grupo A estavam assim distribuidas quanto à idade cronológica: 31 com 7 anos, 17 com 8 anos e 2 com 9 anos. No grupo B encontramos 26 crianças com 7 anos, 12 com 8 anos, 8 com 9 anos e 4 com 10 anos. Quanto ao sexo, observamos que não houve variação significativa. Do ponto de vista da idade cronológica, vamos encontrar um número maior de crianças com mais idade no grupo $B$.

Em cada grupo foi estudado: equilíbrio estático, equilibrio dinamico, coordenação apendicular, coordenação tronco-membros, tono muscular, sensibilidade e gnosias, persistência motora, lateralidade e linguagem.

A avaliação psicológica abrangeu dois testes que nos pareceram da maior importância. Com o WISC, estudamos as condições intelectuais de cada criança e observamos o comportamento delas diante de cada função estudada, o que nos permitiu diagnosticar discrepâncias não só entre as áreas verbais e as de performance, como também entre as várias funções de uma mesma área. Com o teste de Bender, estudamos os niveis de percepção visomotora e orientação espacial.

\section{RESULTADOS}

Podem causar surpresa, de início, os dados globais do grupo A, onde encontramos $48 \%$ das crianças com ENE normal e 52\% com alterações. Pensamos, no entanto, que justamente ai reside a importância da pesquisa prospectiva. Estas crianças continuarão sendo seguidas e provàvelmente um número razoável delas, não sendo atendida de forma adequada, encontrará dificuldades escolares nas $3^{\text {a }}$ ou $4^{\text {a }}$ sértes. Sabe-se que, ả medida que os conteúdos programáticos se tornam mais complexos, pode ocorre uma tendéncia maior ao aparecimento das dificuldades específicas do apredizado.

Para os achados de $48 \%$ de ENE normal no grupo A e $14 \%$ de ENE normal no grupo $\mathrm{B}$ fol calculado o $\chi^{2}$, obtendo-se o resultado de 13,56 . Isto mostra que a diferença entre os achados é estatísticamente significativa ao nivel de $1 \%$.

Todas as crianças do grupo A apresentaram linguagem normal. Uma criança do grupo B (caso 71), apresentava dislalias por troca e por supressão, tendo um padrão de desenvolvimento de linguagem menor do que 3 anos e, embora tivesse 7 anos de idade cronológica, apresentava um coeficiente de desenvolvimento (Q.D.) igual a 21. Outra criança do mesmo grupo (caso 87), apresentava dislalias por supressão, tendo um padrāo de desenvolvimento de linguagem de 3 anos aos 9 anos de idade cronológica, tendo, portanto, um Q.D. igual a 33. 
A pesquisa da lateralidade mostrou: no grupo A - - lateralidade direita $74 \%$, lateralidade esquerda $4 \%$, lateralidade cruzada $22 \%$; no grupo B - lateralidade direita $60 \%$, lateralidade esquerda $4 \%$ e lateralidade cruzada $36 \%$.

Houve incidência igual de sinistros nos dois grupos; nosso achado de $4 \%$ neste item corresponde ao que consta na bibliografia revisada. Chama a atenção a frequiência maior de crianças com lateralidade cruzada ou mal estabelecida no grupo B, onde encontramos $36 \%$, para $22 \%$ no grupo A. Pelo cálculo do $\chi^{2}$ obtemos o valor de 4,19 para estes achados, mostrando ser estatisticamente significativa a diferenca entre eles, ao nivel de $5 \%$.

Quanto ao tono muscular, único aspecto do ENE que já se mostrava patológico ao exame tradicional, foram valorizados os achados de hipotonia em $42 \%$ dos casos do grupo B para $22 \%$ dos casos do grupo A, onde o cálculo do $\chi^{2}$, igual a 4,65 revelou diferença estatisticamente significativa ao nível de 5\%. Não mencionamos paratonias que foram encontradas em vários pacientes porque julgamos se tratar de um aspecto controverso, difícil de ser medido, nitidamente relacionado com a emoção e, portanto, variável de acordo com o ambiente, com o examinador e com o estado psicológico do paciente.

A coordenacão apendicular foi a função que mostrou diferenças mais significativas, pois $44 \%$ das crianças do grupo B acertaram as provas para 7 anos, enquanto $76 \%$ do grupo A o fizeram, obtendo-se um $\chi^{2}$ igual a 10,38, estatísticamente significativo ao nivel de $1 \%$. O interesse para estes achados aumenta na medida em que sabemos ser a coordenação apendicular facilmente avaliada, até mesmo pela professora escolar.

A pesquisa da coordenação tronco-membros demonstrou que $88 \%$ das crianças do grupo B para 98\% das crianças do grupo A conseguiram realizar as provas para 7 anos. O $\chi^{2}$ igual a 3.99 revela ser a diferenca estatisticamente significativa ao nivel de $5 \%$.

O equilibrio estático foi outra função através da qual se observou bem a diferença entre os dois grupos, pois $64 \%$ das crianças do grupo B para $90 \%$ das crianças do grupo A realizaram as provas para 7 anos. A diferenca entre os valores encontrados, pelo cálculo do $\chi^{2}$, neste caso igual a 9,59, é estatísticamente signifiactiva ao nivel de $1 \%$.

Por outro lado, $78 \%$ das crianças do grupo B para $98 \%$ das crianças do grupo A alcançaram as provas para 7 anos na pesquisa do equilibrio dinàmico, o que revelou $\chi^{2}$ igul a 9,57 , com significação estatística para $1 \%$.

Quanto à sensibilidade e gnosias, observamos que $64 \%$ das crianças do grupo B E $74 \%$ das do grupo A não tinham noção adequada de direita e esquerda, sendo esta prova a que mostrou resultados mais semelhantes, com $\chi^{2}=1,22$ não tendo pois, a diferença entre os achados, significado estatístico para os niveis de $1 \%$ ou 5\%. Pensamos ser esta uma funcão na qual está envolvido um aspecto cultural importante.

O estudo da persistência motora revelou que $76 \%$ das crianças do grupo $\mathrm{B}$ para $90 \%$ das criancas do grupo A realizaram as provas para 7 anos. No cálculo do $\chi \%$, obtendo um valor igual a 3,55, concluímos que esta diferença não tem valor estatístico se considerarmos os níveis de significância já citados, embora a simples observação mostre diferenças nitidas.

Estes percentuais se tornam mais significativos se for realizada a correcão de acordo com a idade cronológica. Por este motivo tentamos verificar o coeficiente de desenvolvimento de cada pacicnte e para cada função. Isto só foi possível fazer para os casos em que havia alteração do ENE, pois os que alcancaram as provas para 7 anos não puderam ser considerados uma vez que não sabemos se acertariam provas mais elaboradas. O quadro 1 mostra os resultados ebtidos. 


\begin{tabular}{|c|c|c|c|c|c|c|c|c|}
\hline \multirow{3}{*}{ Função } & \multicolumn{8}{|c|}{ Coeflciente de Desenvolvimento } \\
\hline & \multicolumn{2}{|c|}{$\begin{array}{c}100 \\
\text { Grupo }\end{array}$} & \multicolumn{2}{|c|}{$\begin{array}{c}80-99 \\
\text { Grupo }\end{array}$} & \multicolumn{2}{|c|}{$\begin{array}{l}60-79 \\
\text { Grupo }\end{array}$} & \multicolumn{2}{|c|}{$\begin{array}{l}40-59 \\
\text { Grupo }\end{array}$} \\
\hline & $\overline{\mathbf{A}}$ & $\bar{B}$ & $\overline{\mathbf{A}}$ & $\bar{B}$ & $\mathbf{A}$ & $\mathbf{B}$ & $\overline{\mathbf{A}}$ & $\bar{B}$ \\
\hline Equilíbrio estático & $90 \%$ & $64 \%$ & $4 \%$ & $10 \%$ & $4 \%$ & $18 \%$ & $2 \%$ & $8 \%$ \\
\hline Equilíbrio dinâmico & $98 \%$ & $78 \%$ & $0 \%$ & $6 \%$ & $2 \%$ & $12 \%$ & $0 \%$ & $4 \%$ \\
\hline Coordenação apendicular & $76 \%$ & $44 \%$ & $8 \%$ & $22 \%$ & $16 \%$ & $20 \%$ & $0 \%$ & $14 \%$ \\
\hline Coordenação tronco-membros & $98 \%$ & $88 \%$ & $2 \%$ & $0 \%$ & $0 \%$ & $8 \%$ & $0 \%$ & $4 \%$ \\
\hline Persistência motora & $90 \%$ & $76 \%$ & $0 \%$ & $0 \%$ & $8 \%$ & $14 \%$ & $2 \%$ & $10 \%$ \\
\hline Sensibilidade e gnosias & $74 \%$ & $64 \%$ & $0 \%$ & $0 \%$ & $24 \%$ & $30 \%$ & $2 \%$ & $6 \%$ \\
\hline
\end{tabular}

Quadro 1 - Comparaçio dos coeficientes de desenvolvimento de cada função estudada, nos dois grupos.

A avaliação psicológica pelo WISC permitiu-nos observar que no grupo B estão relacionadas 3 crianças deficientes, 15 "borderline", 30 com Q.I. médio e 2 com Q.I. superior. No grupo A estão relacionadas: 1 criança "borderline", 47 com Q.I. médio e 2 com Q.I. superior.

Traçamos a curva média do WISC em cada grupo e fizemos o estudo comparativo, encontrando resultados semelhantes nas áreas verbais e mais baixos nas áreas de performance no grupo B.

Após a avaliacão psicológica, procuramos integrar os resultados. Como não é possivel apresentar os gráficos do teste de Bender, do WISC e do ENE de cada caso, trazemos os seguintes exemplos a titulo de ilustração:

1. Caso 5 - Criança do grupo A, com 7 anos e 4 meses, WISC superior, Bender normal e ENE normal. Este ê um caso de criança padrão (Figs. 1, 2 e 3).

2. Caso 8 - Crianca do grupo B, com 7 anos e 4 meses, WISC superior, Bender mostrando falhas na orientação espacial e ENE com equilibrio estático, equilíbrio dinâmico, coordenação tronco-membros e persistência motora normais, mas com coordenaçăo apendicular e sensibilidade e gnosias em nivel de 5 anos. A discrepâncía entre os achados mostra que a dificuldade escolar neste caso se prende a disfunções psiconeurológicas (Figs. 4, 5 e 6).

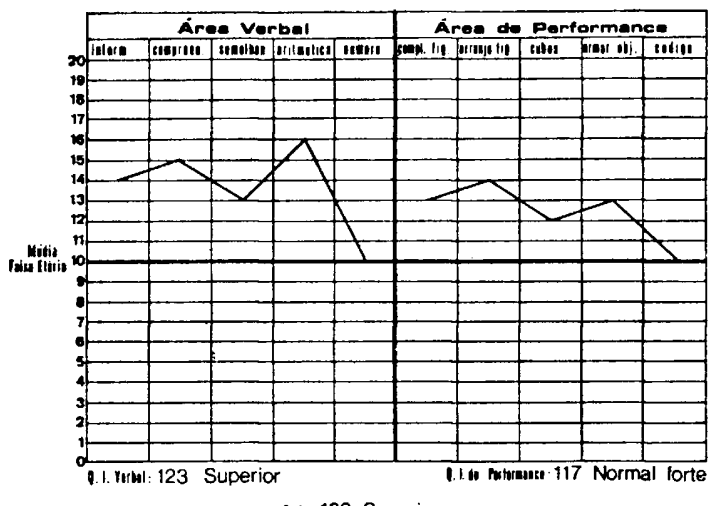

1.1. : 122 Superior

Gráfico 1 - Caso 5. A.P.B. criança do grupo $A$, com 7 anos $e 4$ meses, considerando o caso padrão WISC e mostrando Q.I. superior. 


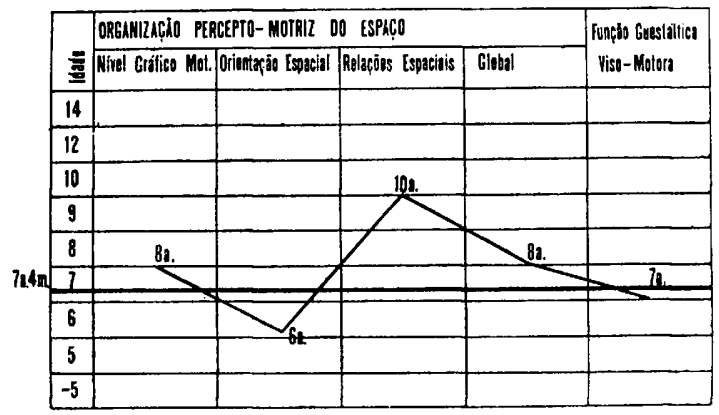

Gráfico Z - Bender da criança padrüo A.P.B. caso 5.

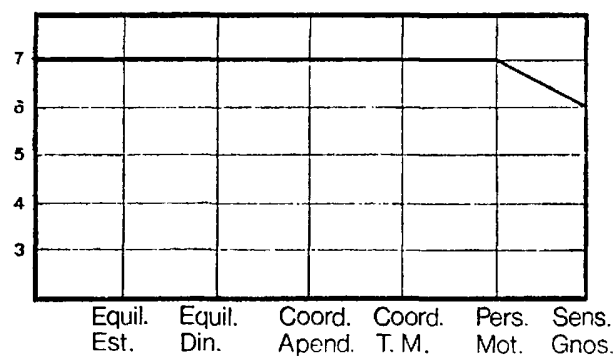

Gráfico 3 - Perfil neurológico da A.P.B. caso 5, criança padrio onde observamos a curva normal ao exame neurológico evolutivo.



Gráfico 4-Caso 8. C.A.A.M., criança do grupo B, com 7 anos $e$ 4 meses. WISC mostrando Q.I. superior. Caso usado para exemplificar situações de dificuldade escolar em crianca com bom nivel intelectual. 


\begin{tabular}{|c|c|c|c|c|c|}
\hline \multirow[b]{2}{*}{ 푤 } & \multicolumn{4}{|c|}{ ORGANILLCTOO PERCEPTO - MOTRAL DO ESPRCTO } & \multirow{2}{*}{$\begin{array}{l}\text { Funglo Gustiflice } \\
\text { Yiso-Molss }\end{array}$} \\
\hline & Nival Gritico & Orimantegin Espreigl & RRibrois Espacilis & Globsl & \\
\hline 14 & & & & & \\
\hline 12 & & & & & \\
\hline 10 & ba. & & & & \\
\hline 9 & & & 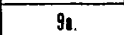 & & \\
\hline 8 & & & $\gamma$ & & -89 \\
\hline 1 & & & Z & & \\
\hline 6 & & & & & \\
\hline 5 & & 58 & & & \\
\hline-5 & & & & & \\
\hline
\end{tabular}

Gráfico 5 - Bender de C.A.A.M. caso 8, mostrando discrepancias entre as areas estudadas.

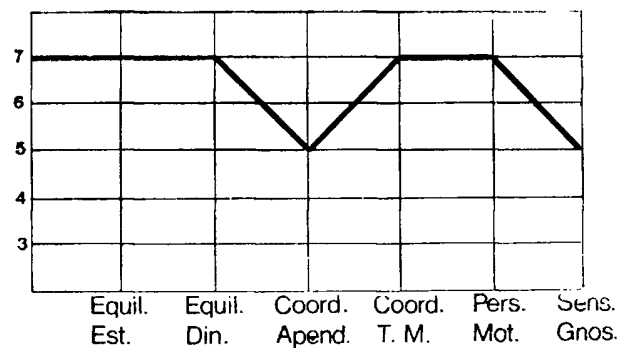

Gráfico 6 - Perfil neurológico de C.A.A.M. caso 8, onde observamos curva caracteristica de disfuncĩo cerebral minima.

\section{CONCLUSOES}

Os objetivos principais deste trabalho foram demonstrar a validade do ENE como mais um método para o diagnóstico das dificuldades do aprendizado, ressaltar a importância do trabalho interdisciplinar e apontar possibilidades terapêuticas. A medida que desenvolvemos o trabalho, fomos acrescentando conclusões que, distribuídas ao lado dos dados numéricos, tornam menos árida esta leitura.

Os dados permitem concluir que: 1) em uma população de crianças matriculadas na $1^{*}$ série do $1^{\circ}$ ciclo, encontramos um número razoável com dificuldades para o aprendizado; 2) dentre estas crianças observamos que as dificuldades para o aprendizado não são sempre proporcionais ao potencial intelectual; 3) na comparação destas crianças com as que apresentavam bom rendimento escolar, encontramos diferentes resultados ao exame neurológico evolutivo no qual foram estudados o equilíbrio estático, o equilíbrio dinâmico, a coordenação apendicular, a coordenação tronco-membros, o tono muscular, a sensibilidade e gnosias, a persistência motora, a lateralidade e a linguagem; 4) a discrepância entre os resultados se prende à maior incidência de sintomas relacionados com disfunção psiconeurológica no grupo que apresenta dificulda- 
des escolares; 5) é possível demonstrar uma correlação entre os achados neurológicos e psicológicos, o que reforça a importância do trabalho em equipe multidisciplinar; 6) o diagnóstico precoce das dificuldades escolares permite o tratamento adequado das diversas síndromes, o que terá grande valor na redução das estatísticas de reprovação, evasão escolar e marginalização do menor.

Nosso trabalho inicia no momento em que todos os aspectos aqui referidos forem devidamente valorizados e a observação seguida e ampliada, com o fim de melhorar as cond:ções do escolar. Acreditamos que isto só será possível na medida em que seja desenvolvido um plano global de assistência ao eđucando, centralizado na investigação neurológica e neuropsicológica.

\section{RESUMO}

São estudados, de forma comparativa, dois grupos de 50 crianças cada um, com bom e com mau rendimento escolar. As 100 crianças, entre 7 e 10 anos de idade, que em 1973 cursaram a $1^{\prime \prime}$ série do $1^{\circ}$ Ciclo, foram submetidas a exame neurológico evolutivo, testes de Bender e WISC. O tratamento estatístico dos achados permite concluir pela importância da avaliação e do atendimento neurológico e neuropsicológico nas dificuldades para o aprendizado.

\section{SUMMARY}

\section{Neurologic evolutive exumination und upprenticeship difficulties}

A comparative study betwen two groups of fifty children, whose ages ranged from 7 to 10 years old and presented, respectively, good and poor development in class in 1973, is reported. The scholars were evaluated by the evolutive neurological examination, WISC and Bender Test. By statistical analysis it is emphasized the importance of the neurological and neuropsychological evaluation and management of scholar difficulties.

\section{REFERENCTAS}

1. AJURIAguerra, J, de - Vue d'ensemble sur les troubles d'evolution de la motricité, du language et du caractère, a disfonctionemment conjoint. Sauv. de l'Enfance (Paris) 28:3, 1949.

2. BELMONT, L. \& BIRCH, H. J. - Lateral dominance and right-left awareness in normal children. Child Development 34:257, 1963.

3. BENDER, L. - Test Guestaltico Visomotor. Editorial Paidós, Buenos Aires, 1964.

4. CLEMENTS, S. D. \& PETERS, J. E. - Minimal brain disfunctions in the school age child. Arch. Gen. Psychiat. 6:185, 1962.

5. DIAMENT, A. J. - Contribuição para a sistematização do exame neurológico de crianças normais no primeiro ano de vida. Tese, Faculdade de Medicina da Universidade de São Paulo, 1969.

6. GESELL, A. \& AMATRUDA, C. - Diagnostico del Desarrollo Normal y Anormal del Niño. Versão castelhana. Editorial Paidós, Buenos Aires, 1946.

7. KNOBLOCH, H. \& PASAMANICK, B. - The developmental behavioural approach to the neurologic examination in infance. Child. Develop. 33:181, 1962. 
8. LEFEVRE, A. B. - Contribuição para a padronização do exame neurológico do recém-nascido normal. Tese, Faculdade de Medicina da Universidade de São Paulo, 1972.

9. LEFìve, A. B. - Exame Neurológico Evolutivo. Ed. Sarvier, São Paulo, 1972.

10. MORAES ALMEIDA, R. - Lateralidade, maturidade para leitura e escrita e rendimento escolar de canhotos e destros. Tese de doutoramento, Faculdade de Filosofia da Universidade de São Paulo, 1965.

11. POPPOVIC, A. M. - Alfabetização e Disfunçōes Psico-Neurológicas. Editora Vetor, São Paulo, 1968.

12. ROTTA, N. T. - Desenvolvimento psicomotor. Pesquisa Médica 9:617, 1973.

13. TOWEN, B. C. L. \& PRECHTL, H. F. R. - The neurological examination of the child with minor nervous dysfunction. Spastics Inter. Med. Publ., London, 1970.

14. WECHSLER, D. - Weschsler Inteligence Scale for Children. The Psycol. Corp., New York, 1949.

Rua Mostardeiro 291, conj. 405 - 90000 Porto Alegre, RS - Brasil 